\title{
BGP routing design and optimization of large scale network
}

\author{
Liu Lang \\ Mathematics and Information Engineering Department of Jingdezhen College, Jiangxi Jingdezhen \\ 333000, China
}

Keywords: BGP, routing strategy, routing optimization

\begin{abstract}
The BGP routing design concept and the configuration data are proposed in this paper, on the basis of this network, the routing optimization in three aspects is taken, and the optimization idea and configuration data are presented.
\end{abstract}

\section{Introduction}

The large internal network is combined by 3 routers as RT1\RT2\RT3, the 3 routers belongs to AS600, R2 and R3 are the border routers of AS, in order to enhance the reliability, they are respectively connected with the ISP-1 (AS500 RT4) and ISP-2 (AS400 RT5). Because there are two exported, it is necessary to implement the load sharing processing. Based on the above consideration[1-3], we determine the BGP are deployed between the local network and ISP, realize the reliability of the BGP dynamic routing protocol with backup function, the powerful BGP attributes are used to control the routing, and achieve load sharing. At the same time, the route strategy is used to realize the more powerful routing control and routing filtering. The internal network takes the IGP routing design, it is completed by OSPF[4-6]. Network topology structure is shown in figure 1.

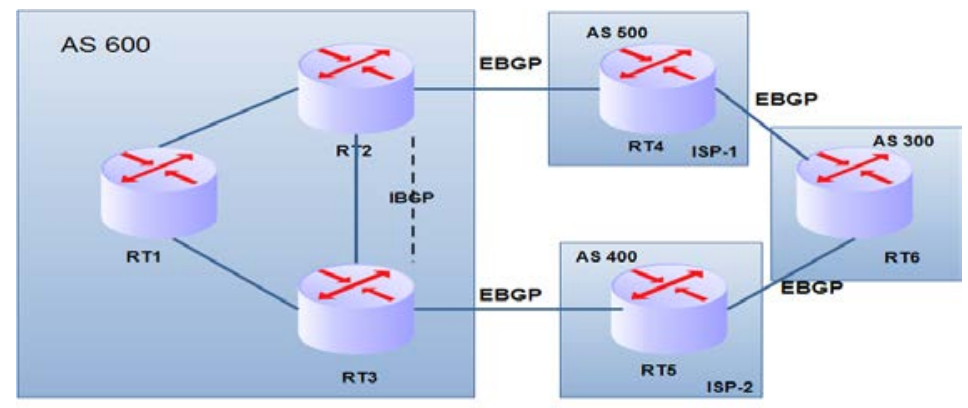

Figure 1 Network topology structure

\section{Principle of network design}

A Data configuration

The data configuration results are expressed in Table 1 .

\begin{tabular}{|l|l|l|l|l|}
\multicolumn{5}{|c|}{ Table 1 Data configuration results } \\
\hline RT1 & Loopback0 & Eth1/0/1 & Eth1/0/2 & \\
& 1.1 .1 .1 & 12.12 .12 .1 & 13.13 .13 .1 & \\
\hline RT2 & Loopback0 & Eth0/0 & Eth0/1 & S1/0 \\
& 2.2 .2 .2 & 12.12 .12 .2 & 23.23 .23 .2 & 24.24 .24 .2 \\
\hline RT3 & Loopback0 & GigEth0/0 & GigEth0/1 & S6/3 \\
& 3.3 .3 .3 & 13.13 .13 .3 & 23.23 .23 .3 & 35.35 .35 .3 \\
\hline RT4 & Loopback0 & Eth0/0 & & S2/0 \\
& 4.4 .4 .4 & 46.46 .46 .4 & & 24.24 .24 .4 \\
\hline RT5 & Loopback0 & GigEth0/0 & & S6/0 \\
& 5.5 .5 .5 & 56.56 .56 .5 & & 35.35 .35 .5 \\
\hline RT6 & Loopback0 & Eth1/0/1 & Eth1/0/2 & Loopback1 \\
& 6.6.6.6 & 46.46 .46 .6 & 56.56 .56 .6 & 100.100 .100 .100 \\
\hline
\end{tabular}


B R1 design for internal router R1open

The OSPF protocol and corresponding segment is developed, data are shown as follows:

[r1] ospf 1

area 0.0 .0 .0

network 1.1.1.1 0.0.0.0

network 13.13.13.0 0.0.0.255

network 12.12.12.0 0.0.0.255

C R2 is an autonomous system boundary router, BGP and OSPF routing protocol are enabled, the corresponding segment is released, the BGP peer is configured, the data are shown as follows:

[r2]bgp 600

network 1.1.1.1 255.255.255.255

network 2.2.2.2 255.255.255.255

network 12.12.12.0 255.255.255.0

network 23.23.23.0 255.255.255.0

network 24.24.24.0 255.255.255.0

undo synchronization

peer 24.24.24.4 as-number 500

peer 3.3.3.3 as-number 600

peer 24.24.24.4 route-policy cl import

peer 3.3.3.3 next-hop-local

peer 3.3.3.3 connect-interface LoopBack0

ospf 1

area 0.0 .0 .0

network 12.12.12.0 0.0.0.255

network 23.23.23.0 0.0.0.255

network 2.2.2.2 0.0.0.0

D R3is referred as an autonomous system boundary router, enabling BGP and OSPF routing protocol, the corresponding segment is released and the BGP peer is configured, data are shown as follows:

[r3]bgp 600

network 1.1.1.1 255.255.255.255

network 3.3.3.3 255.255.255.255

network 13.13.13.0 255.255.255.0

network 23.23.23.0 255.255.255.0

network 35.35.35.0 255.255.255.0

undo synchronization

peer 2.2.2.2 as-number 600

peer 35.35.35.5 as-number 400

peer 2.2.2.2 next-hop-local

peer 2.2.2.2 connect-interface LoopBack0

peer 35.35.35.5 r

area 0.0 .0 .0

network 13.13.13.0 0.0.0.255

network 23.23.23.0 0.0.0.255

network 3.3.3.3 0.0.0.0

E R4 is referred as AS500 internal routing, and the corresponding peer relationship is established, data are shown as follows:

[r4]bgp 500

network 4.4.4.4 255.255.255.255

network 24.24.24.0 255.255.255.0

network 46.46.46.0 255.255.255.0

undo synchronization 
peer 24.24.24.2 as-number 600

peer 46.46.46.6 as-number 300

F R5 is referred as AS400 internal routing, and the corresponding peer relationship is established, data are shown as follows:

[r5]bgp 400

network 5.5.5.5 255.255.255.255

network 35.35.35.0 255.255.255.0

network 56.56.56.0 255.255.255.0

undo synchronization

peer 56.56.56.6 as-number 300

peer 35.35.35.3 as-number 600

G R6 is referred as AS300 internal routing, and the corresponding peer relationship is established, data are shown as follows:

[r6]bgp 300

aggregate 100.100.0.0 255.255.0.0 detail-suppressed

network 6.6.6.6 255.255.255.255

network 46.46.46.0 255.255.255.0

network 56.56.56.0 255.255.255.0

network 100.100.100.100 255.255.255.255

undo synchronization

peer 56.56.56.5 as-number 400

peer 46.46.46.4 as-number 500

peer 46.46.46.4

peer 46.46.46.4

\section{Network optimization design}

R1 connectivity problem.According to the basic design, R2 $\backslash R 3 \backslash R 4 \backslash R 5 \backslash R 6$ can interact for protocol interworking based on BGP, R1\R2 $\backslash \mathrm{R} 3$ can pass the OSPF protocol interworking, but the $\mathrm{R} 1$ cannot communicate with whole network, the reason is that R1 only can clear OSPF routing entry, R2 and R3 cannot introduce BGP routing entry within the OSPF process, through analysis to solve the R1 connectivity problems, R2 and R3 release the default routing in the OSPF domain, and set the R2 default routing, when the overhead value is 100 less than the R3 of the cost value, he R1 will take ISP1 R3 as the preferred export enterprise network.

(1) Configuration data are described as follows:

[r2]ospf $1 / / \mathrm{r} 2$ sets the default routing overhead is 100

default-route-advertise always cost 100

[r3] ospf $1 / / / \mathrm{r} 3$ sets the default routing overhead is 200

default-route-advertise always cost 200

(2) The test data are expressed as follows:

$<$ r1 $>$ dis ip routing-table

Destination/Mask Proto Pre Cost NextHop Interface

0.0.0.0/0 O_ASE $150100 \quad 12.12 .12 .2$ Eth1/0/1

(3) Test conclusion is the next hop of R1Router is Eth0/0 ---- 12.12.12.2 of R2.

Direction data distribution and optimization of enterprise network.

(1) Design ideas

The local intranet segment is RT1---RT2---AS50, the direction of RT1 is ISP1(AS 500), access to any other network ,the network path is RT1---RT2---RT3---, it is the objective segment of network.

(2) Configuration data are shown as follows:

1) R2 data

[r2]peer 24.24.24.4 route-policy cl import // The implementation of the CL strategy from the R4 
into the data stream

route-policy cl permit node 10

if-match as-path 100 // If matching as-path 100 (defined later)

apply local-preference 300 //Set the value of local-preference is 300 (The default value is 100)

route-policy cl permit node 20 // Other matching, do nothing

ip as-path 100 permit $\wedge 500 \$ / /$ The regular expression, the AS500 is taken as the source of data

2) R3data

[r3]peer 35.35.35.5 route-policy cl import //The implementation of the CL strategy is taken from the R5 data stream

route-policy cl permit node 10

apply local-preference 200 // All local-preference value of data is 200(The default value is 100)

Test data.

$<$ r2>dis bgp routing-table

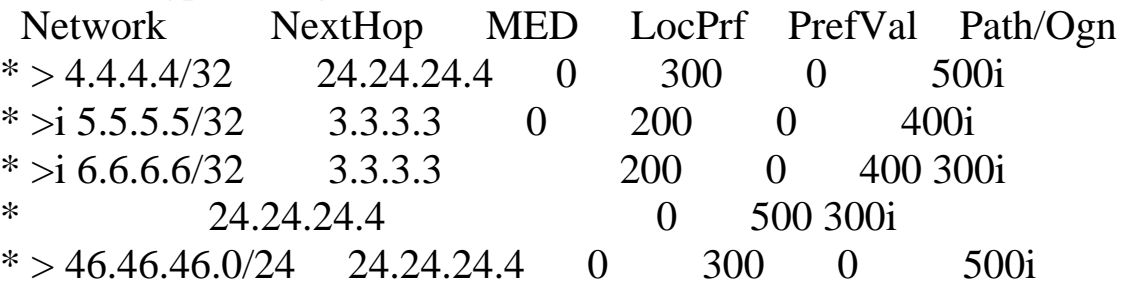

Test conclusions.

From the test data obtained through Ogn (as source), Ogn is the next hop address of 500i, the data is R4 S2/0, the other Ogn is 400i or 300i, its next hop is R3.

\section{Direction data optimization of enterprise network}

\section{Design ideas.}

R4 does not send other AS data to R2. The R6 is configured the community property, and set NO-EXPORT parameters, the R4 will not release R6 data to the R2, but it will pass through R2-R3R5-R6.

Configuration data.

$<\mathrm{r} 6>$ acl number 2000 // To define an access control list, name 2000

rule 0 permit source 6.6.6.6 0 // The IP of the source address Rule 0 is 6.6.6.6

peer 46.46.46.4 route-policy tt export // Data flows carry out TT strategy from R6 to R4

peer 46.46.46.4 advertise-community // Set the group attribute

route-policy tt permit node 10 // Establish TT strategy

if-match acl 2000 // If match the control list 2000

apply community no-export // Executive group properties for the no-export parameter

route-policy tt permit node 20 // The other is matching, do nothing operation

Test data.

[r2]dis bgp routing-table



Test results.

Through the test data as above, obtain that the next hop of AS300 6.6.6.6 is R3, instead of R2.

\section{Conclusions}

Through the design and optimization as above, enterprise network export is RT2, the internal network data of ISP1(AS500) is forwarded by RT2, the other AS data through the RT3 for forwarding, the external data of enterprise is selected as RT3 firstly. 


\section{Acknowledgements}

Jingdezhen Institute of Youth Fund Project:

Campus network wireless hotspot map drawing and security research

\section{References}

[1] Li Feng, Wu Chun- ming. Research on Prevention Fluctuation Control method of Network Intrusion Based on Energy Management[J]. Computer simulation, 2013,30(12): 45-48, 335.

[2] Wang Guang-hao, Wu Yue. Data Trust Model for Road Information in Vehicular Ad hoc Network [J]. Computer Science, 2014,41(6):89-93.

[3] Xu Xiao-bin, Zhang Guang-wei, Sun Qi-bo, et al. Precision Configurable Data Aggregation Algorithm in WSNs[J]. Acta Electronica Sinica, 2014, 42(6):1205-1209.

[4] Luo Liang, Wu Wen-Jun, Zhang Fei. Energy Modeling Based on Cloud Data Center[J]. Journal of Software, 2014,25(7):1371-1387.

[5] Song Minghong, Yu Huafeng, Chen Haiyan. Application of Improved Quantum Evolutionary Algorithm in Computer Network's Routing Choice[J]. Bulletin of Science and Technology, 2014,30(1):170-173. 